\title{
Angiostrongylus vasorum: epidemiological, clinical and histopathological insights
}

\author{
Laura Rinaldi ${ }^{1,2^{*}}$, Laura Cortese ${ }^{1}$, Leonardo Meomartino ${ }^{1}$, Teresa B Pagano ${ }^{1}$, Paola Pepe ${ }^{1}$, Giuseppe Cringoli ${ }^{1,2}$ \\ and Serenella Papparella ${ }^{1}$
}

\begin{abstract}
Background: Canine angiostrongylosis is a nematode infection in domestic dogs and wild carnivores. The present report focuses on epidemiological, clinical and histopathological findings in a case of fatal disseminated angiostrongylosis in a dog living in southern Italy and provides data on the extent of the spread of Angiostrongylus vasorum in the same area.

Case presentation: A 4-year-old female English Setter from the Campania region of southern Italy was referred with a 2-week history of cough and severe respiratory distress that did not respond to antimicrobial therapy. Based on clinical, radiological, echographical and cytological findings (including the presence of larvae), a suspect diagnosis of lungworm infection was performed. After few days the dog died due to progressive clinical aggravation. Complete postmortem examination was conducted within 24 hours from death and samples from lungs, heart, liver, kidney, spleen, stomach and small intestine were fixed in 10\% buffered formalin. Grossly, several hemorrhagic foci were observed mostly in the lungs, liver, kidney. Microscopically, the lungs contained numerous, multifocal to coalescing granulomas composed of epitheliod macrophages, multinucleated giant cells and some neutrophils, frequently associated with parasite eggs and larvae. The lungs contained many firm nodules, many adult nematodes approximately 1.5 to $2 \mathrm{~cm}$ in length were observed in cut sections and identified as A. vasorum. A subsequent parasitological survey performed with FLOTAC on stray dogs living in the same area showed the presence of A. vasorum larvae in 17 of 1639 stray dogs examined (1.04\%).
\end{abstract}

Conclusion: This survey provides new data on distribution of $A$. vasorum and underlines that canine angiostrongylosis should be considered as differential diagnosis in dogs.

Keyword: Angiostrongylus vasorum, Dog, Pathology, Diagnosis

\section{Background}

Angiostrongylus vasorum commonly known as the "French heartworm" is a metastrongyloid nematode of dogs and other canids, including various species of fox, wolf, coyote and jackal (definitive hosts) [1]. The adults reside in the right side of the heart and in the pulmonary arteries, with potentially severe consequences for the host [2]. The infection can cause a wide range of disease outcomes, which are most often characterized by respiratory dysfunction, but it can also manifest as bleeding, neurological, cardiovascular or gastrointestinal disorders, with or without respiratory involvement [3].

\footnotetext{
*Correspondence: Irinaldi@unina.it

${ }^{1}$ Department of Veterinary Medicine and Animal Productions, University of Naples Federico II, Via Della Veterinaria 1, 80137 Naples, Italy

${ }^{2}$ Inter-University Center for Research in Parasitology (CIRPAR), Naples, Italy
}

The geographic distribution of the parasite includes various countries of Europe, North and South America as well as Africa [1]. The parasite appears to be quite common in well-isolated endemic foci, with few occasional reports occurring outside them [4]. However, recent reports challenge this traditional view, as $A$. vasorum has appeared in several new geographical areas [5]. Italy is one of the countries where A. vasorum is undoubtedly spreading, offering ideal environmental and epidemiological conditions for the expansion of A. vasorum and the establishment of further new endemic foci $[6,7]$. The reasons for this emergence are unclear and poorly understood, but may involve global changes leading to spread of a variety of definitive, intermediate and paratenic hosts and consequent modification of mollusk phenology [8]. Due to this spread and the increasing clinical relevance of canine angiostrongylosis, the interest in 
A. vasorum is growing, particularly with respect to its treatment and control [9].

At present, diagnosis relies on clinical manifestations, diagnostic imaging, bronchial washings and on the detection of the first-stage larvae (L1) in faecal samples. The parasite detection in faecal samples is usually performed using conventional coprological examination such as direct faecal smears, flotation and Baermann (gold standard) [10]. Also, the FLOTAC techniques [11] have been demonstrated as very sensitive for the diagnosis of $A$. vasorum in dogs [12]. Furthermore, serological [13,14] and molecular $[15,16]$ methods have been developed for the diagnosis of canine angiostrongylosis.

With the aim to provide further insights on this parasite, the present paper describes post mortem gross and histopathological observation in a dog with a fatal infection by $A$. vasorum. Subsequently to this case, a coprological survey was performed to evaluate the extent of $A$. vasorum infection in stray dogs living in the city of Naples (southern Italy), using the FLOTAC techniques.

\section{Case presentation}

A 4-year-old female English Setter from the Campania region of southern Italy was referred with a 2-weeks history of cough and severe respiratory distress that did not respond to antimicrobial therapy. On physical examination, the dog was alert, dyspneic and tachypneic. Mucous membranes and capillary refill time were normal. Cardiac auscultation revealed tachycardia.

Hematological abnormalities included anemia and leukocytosis. Routine biochemical analyses were unremarkable. An electrocardiogram demonstrated right axis deviation. Thoracic radiographs in standard lateral-lateral projection and toracic ultrasound were carried out and an ultrasoundguided fine needle aspiration was performed on selected pleural lesions. Cytological evaluation showed mixed inflammatory cells occasionally admixed with full nematode larvae. Based on clinical, radiological, ultra sonographical and cytological findings (including the presence of larvae), a suspect diagnosis of lungworm infection was made; however, no larvae were detected in faecal samples using the Baermann technique [17]. A therapeutic protocol including antibiotics, corticosteroid and milbemycin oxime was administered. Ten days later the dog was hospitalized following a severe pneumothorax. Supplemental oxygen therapy was administered and a chest tube was placed. Subsequently pneumothorax developed again; thoracic surgery was necessary and led to its resolution. One month after surgery, the dyspnea worsened. After a few days, the dog suddenly died despite supplemental oxygen therapy. Complete postmortem examination was carried out within 24 hours of the death and samples from lungs, heart, liver, kidney, spleen, stomach and small intestine were fixed in $10 \%$ buffered formalin. Pulmonary arteries were opened and examined in order to identify intravascular nematodes. Some cytological smears were performed from tracheobronchial lymphnode. Many adult worms were collected and morphologically identified as adult $A$. vasorum based on morphology of the adult male bursa and the length of the spicules [18]. Formalin-fixed samples were processed for sectioning, embedded in paraffin, sectioned at 5 micron, and stained with hematoxylin and eosin (HE), and Periodic Acid Shiff (PAS) stain.

\section{Radiological findings}

Thoracic radiographs showed a multifocal to coalescing bronchial and alveolar pattern and enlargement of tracheobronchial lymph nodes (Figure 1). Thoracic ultrasound showed multiple sub pleural nodules (Figure 2) from which an ultrasound-guided fine needle aspiration was performed. B-mode echocardiography showed a mild dilation of the right atrial, main pulmonary trunk and right pulmonary artery. Doppler echocardiography revealed tricuspid regurgitation (maximal velocity: $243 \mathrm{~cm} / \mathrm{s}$; maximal pressure gradient, $23,6 \mathrm{mmHg}$ ).

\section{Pathological findings}

Macroscopically, pathological findings of the lungs consisted in multiple, poorly defined dark-red foci of necrotichemorrhagic areas. Firmer areas of broncopneumonia were also present. Several adult parasites, approximately 2$2.5 \mathrm{~cm}$ in length, were collected from pulmonary arteries (Figure 3). Alveolar septa were mildly expanded with an increased amount of dense fibrous tissue. The overlying pleura were moderately thickened too.

Microscopically, large areas of pulmonary parenchyma were replaced by numerous, multifocal to coalescing granulomas composed of macrophages, multinucleated giant cells, eosinophils and neutrophils granulocytes. The center of these granulomas contained unembryonated and embryonated parasite eggs, coiled larvae and deposits of amorphous, pale, eosinophilic necrotic debris, while lymphocytes and plasma cells were present at the periphery (Figure 4). Fibroblastic proliferation with septal thickening and lymphoid hyperplasia were also observed. Numerous organized thrombi (Figure 5), often containing large adult nematodes were present in lumen of medium-sized pulmonary arteries, causing eosinophilic vasculitis. Occasionally, adult parasites were seen within the alveoli, which were affected by productive alveolitis with cell esfoliation and presence of luminal suppurative exudate. Wide hemorrhagic areas associated with hemosiderin deposits, hemosiderin-laden macrophages, coagulative necrosis foci, edema and hypersecrection of mucous glands where found in the remaining parenchyma.

Many adult worms were found in right ventricular cavity. Macroscopically, the right ventricular wall was thickened and trabeculae carnae muscles were hypertrophied. 


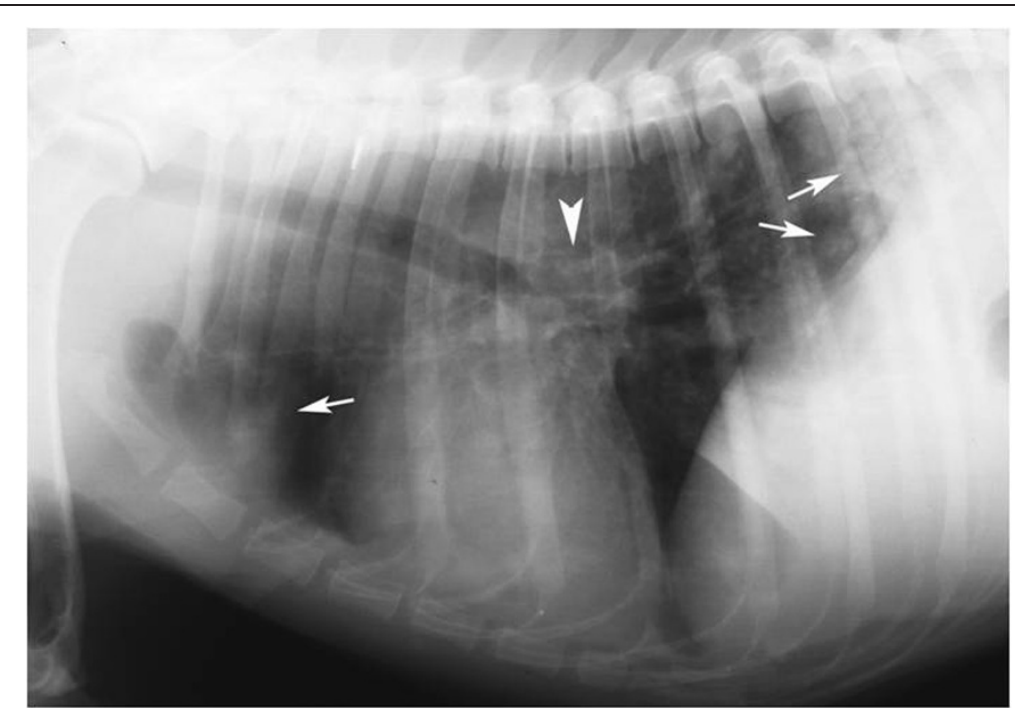

Figure 1 Lateral radiograph on right recumbency: there is a diffuse opacification on lung fields with a mixed nodular interstitial, bronchial and alveolar pattern (arrows); tracheobronchial lymph nodes are enlarged (arrowhead).

The whole heart appeared moderately enlarged and rounded.

Microscopic myocardial injuries resulted from aberrant larval migration; multifocally, granulomatous foci mainly centered around parasite eggs or larvae were detected, disrupting the normal myocardial architecture. Multiple foci of necrosis of cardiomyocytes associated to interstitial edema and infiltration of eosinophils was observed as well. Occasionally, adult worms were found in vessel lumen, inducing vasculitis and perivascular inflammation
(Figure 6). Grossly moderate bilateral enlargement of the kidneys was observed; radial, whitish striae were evident on the cut surface (Figure 7).

Histologically, several renal injuries were present. PAS stain demonstrated membranoproliferative glomerulonephritis with apparent duplication of glomerular basament membrane (tram-track appearance) was observed (Figure 8), thickening of Bowman's capsule and glomerular mesangial matrix deposits. Glomerular atrophy, thrombi and larvae within the glomerular capillaries were also reported. Other

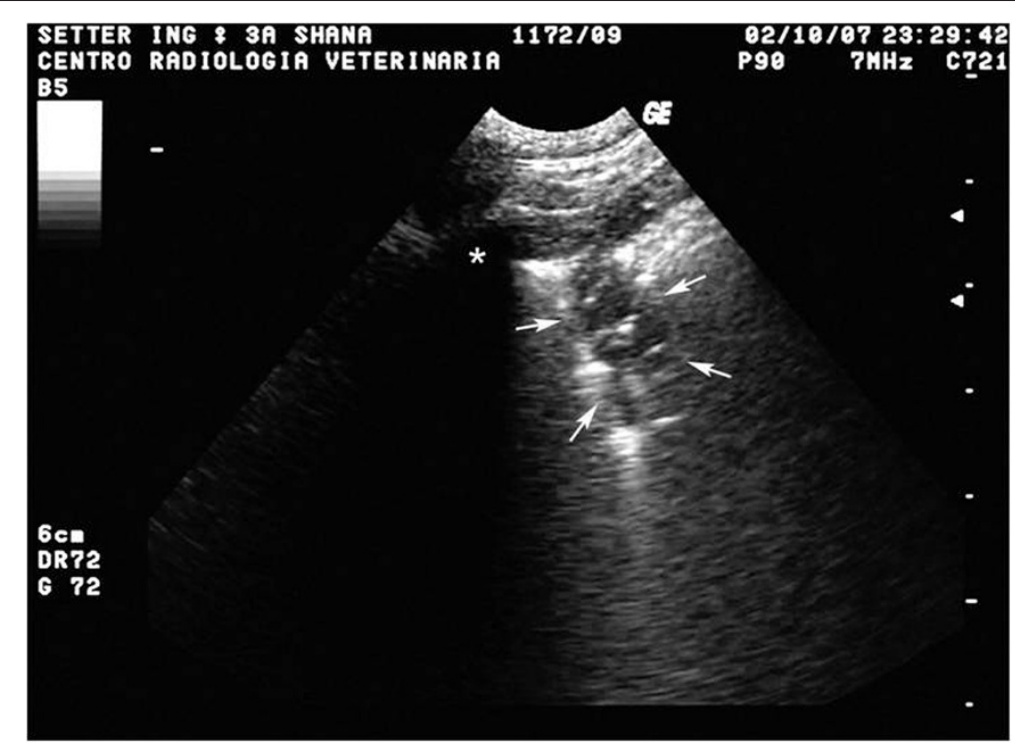

Figure 2 Ultrasound dorsal longitudinal scan trough the 9th left intercostal space: a sub pleural hypoecoic nodule is visible (arrows); residual air in alveoli and bronchioles appears as hyperecoic speckles inside the nodule; on the left side there is a rib acoustic shadowing (asterisk). 


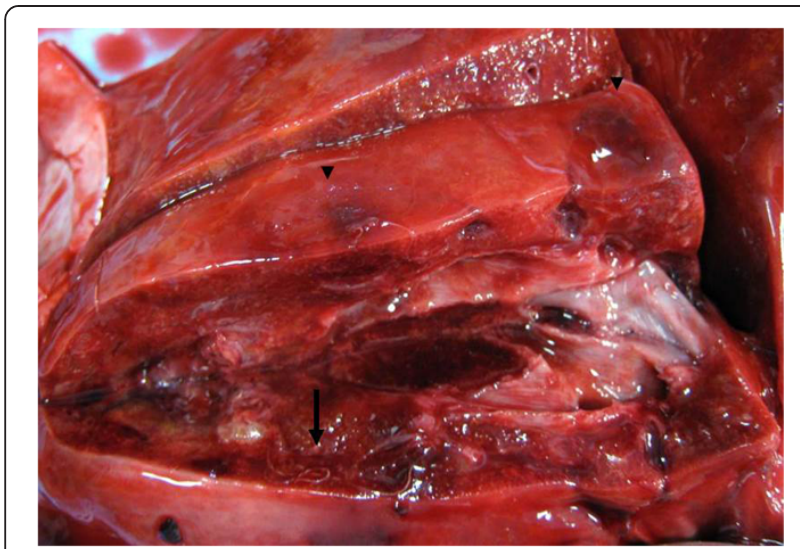

Figure 3 Lung. Pulmonary inflammation and fibrosis. Note the presence of necrotic hemorrhagic areas (arrowheads) and some larvae (arrow).

histopathological findings included hyaline droplet nephropathy, interstitial inflammatory infiltrates, and hemorrhages.

In the liver numerous single, disseminated inflammatory elements, consisting of lymphocytes, plasma cells and eosinophil granulocytes were observed either in the portobiliar spaces or around centrolobular veins. Some small granulomas containing the remains of larvae were also present. The remaining hepatic parenchyma showed moderate interstitial hemorrhages, deposits of bile pigments and focal hyperplasia of Kupffer's cells.

The superficial mucosa and the deep lamina propria of the stomach showed several clusters of mononuclear inflammatory cells admixed with eosinophil granulocytes. Sometimes the inflammatory infiltrate was perivascular.

Signs of eosinophilic enteritis, mostly involving the small intestine, were observed. They included lymphoid hyperplasia, presence of numerous eosinophil granulocytes and mononucleated inflammatory cells in the axis of the villi

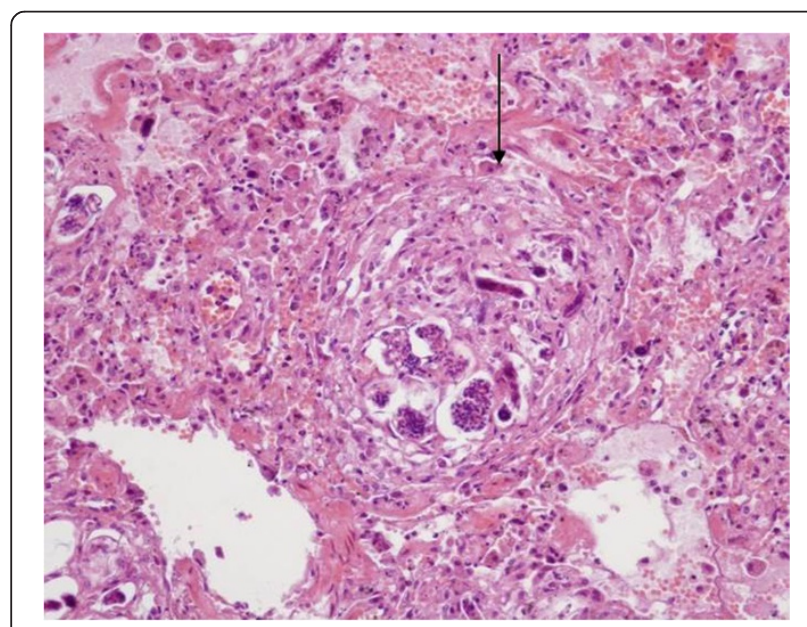

Figure 4 Lung. Pulmonary granuloma (arrow). Some unembryonated and embryonated parasite eggs, coiled larvae and cellular debris are visible in the center. $\mathrm{H}$ \& E stain (20X).

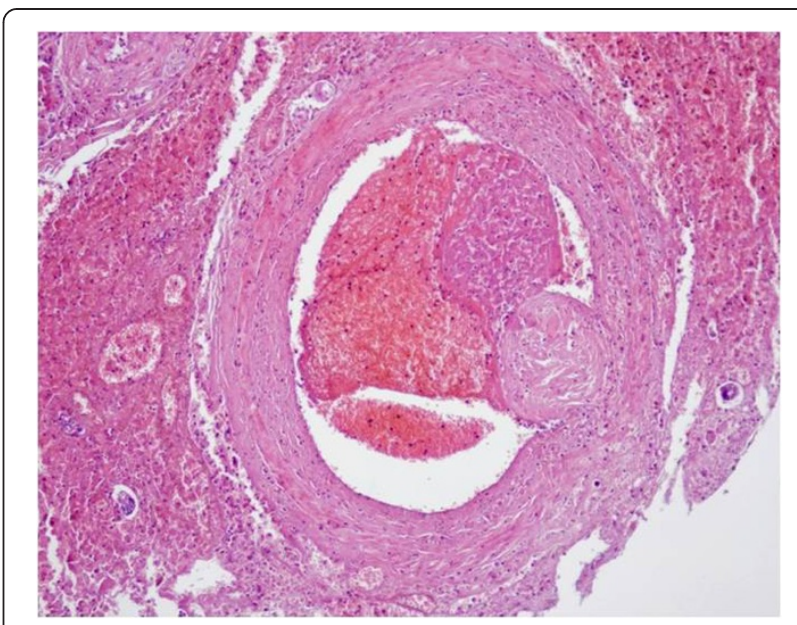

Figure $\mathbf{5}$ Lung. Large organized thrombus in lumen of an artery. $H \& E$ stain $(10 x)$

and hypersecrection with cystic aspects of the mucous glands. This inflammation remained confined at the lamina propria level, without involving the lower layers of the intestinal wall.

Microscopically, the pancreas showed interstitial hemorrhages, focal eosinophilic inflammatory infiltrates and some small granulomas, which contained nematode eggs or larval remains.

The spleen were affected by a severe lymphocytic depletion, while the connectival septa and the serosa appeared very thickened.

\section{Coprological survey}

Between January 2012 and December 2013 faecal samples were routinely collected from 1639 stray dogs living in the

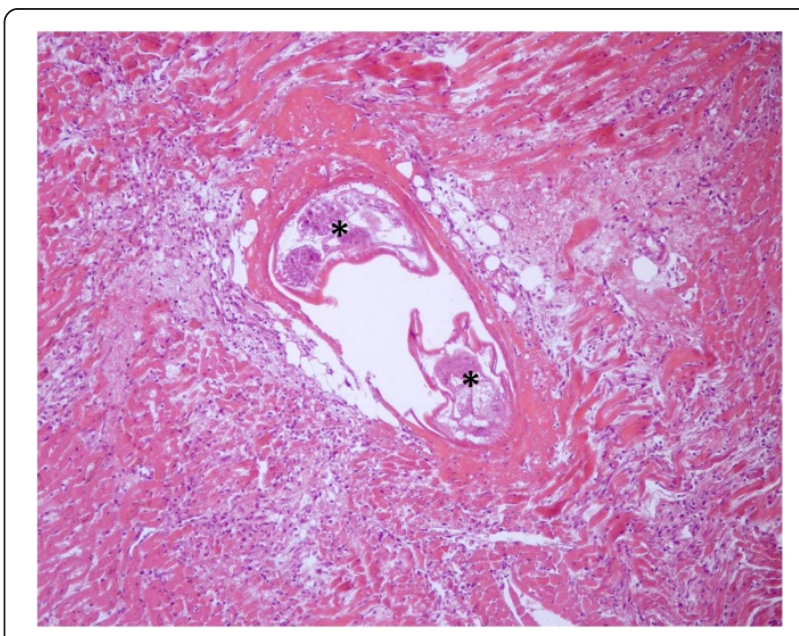

Figure 6 Heart. Adult worms in vessel lumen (asterisks), inducing vasculitis and perivascular inflammation. Note also the coagulative necrosis of the cardiomyocytes and interstitial myocarditis. $\mathrm{H}$ \& E stain $(10 \times)$. 


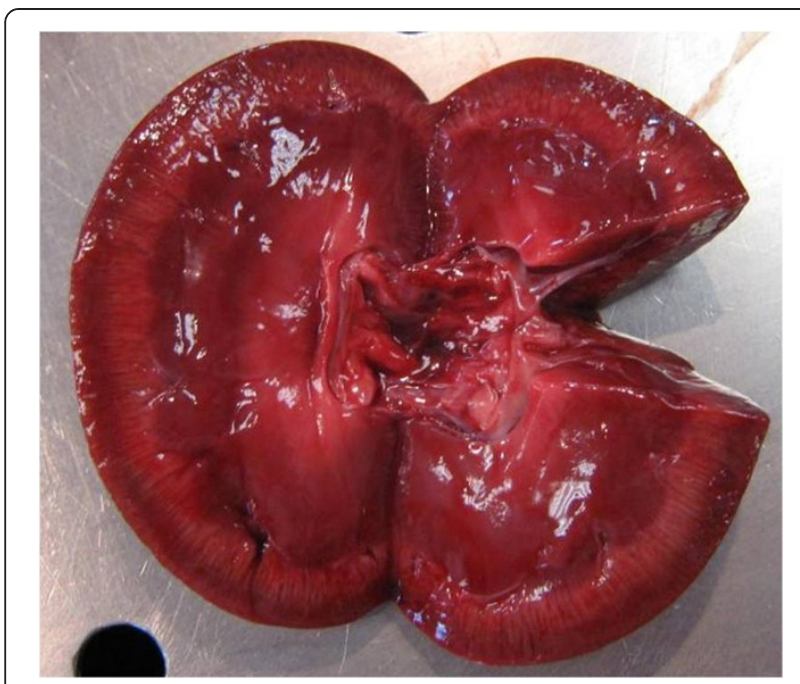

Figure 7 Kidney. Interstitial nephritis.

city of Naples (Campania region, southern Italy) and brought to the veterinary hospital of the Department of Veterinary Medicine for sterilization. Information regarding sex, age and clinical signs was collected at the time of arrival to the veterinary hospital. The age of each animal was recorded based on dental examination. A minimum of $2 \mathrm{~g}$ of faeces was collected from each animal, immediately placed into a container, and fixed 1:4 with formalin $5 \%$ before being analyzed.

Each sample was examined by the FLOTAC basic technique [12] (using zinc sulphate s.g. 1.20 as flotation solution). A differential diagnosis was also performed to discriminate A. vasorum first stage larvae (L1) from those of Crenosoma vulpis, Oslerus osleri or Filaroides spp. on the basis of morphological finding at the tail according to

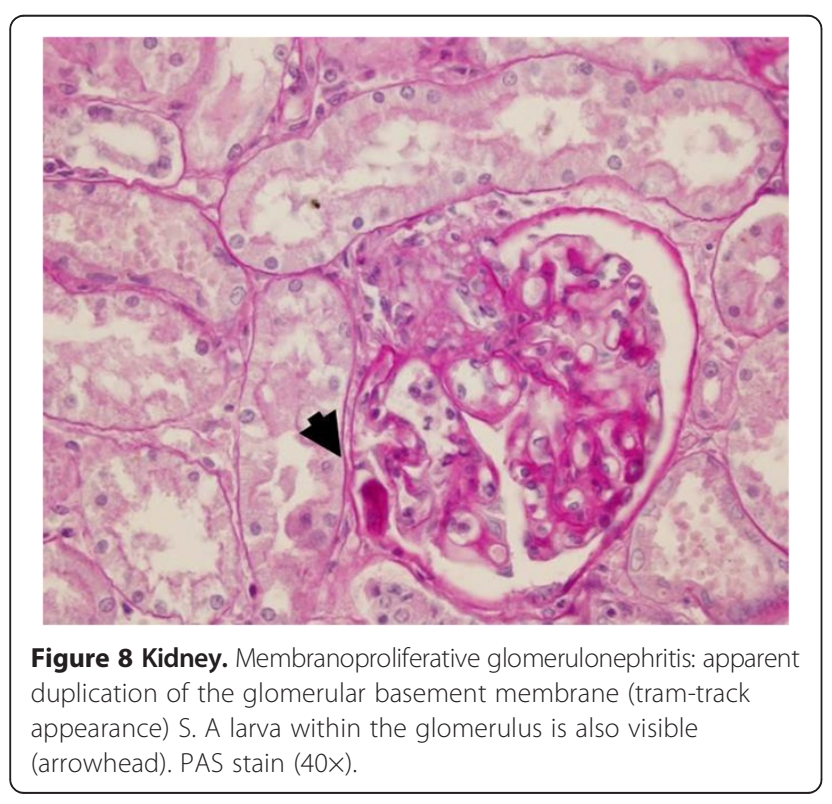

Helm et al. [19]: the tapered tip of the tail of A. vasorum L1 has a kink with a dorsal spine (Figure 9).

The presence of $A$. vasorum was detected in 17 of 1639 stray dogs examined (1.04\%; 95\% CI $=0.63-1.69)$. Among the others lungworms, only $\mathrm{O}$. osleri was detected in 2 of the 1639 dogs examined $(0.12 \%$; $95 \% \mathrm{CI}=0.02-0.49)$.

\section{Conclusions}

This report documents a case of fatal canine angiostrongylosis in southern Italy. The clinical, pathological, and parasitological findings are consistent with a severe and diffuse angiostrongylosis. In fact, the clinical manifestations associated with this disease may vary greatly from subclinical state (with no or minor sign) to fatal condition. The cardiorespiratory distress due to the inflammatory response to eggs and migrating larvae [20,21], bleeding disorder such as hemorrhagic diatheses and coagulation defects, cardiac failure and other nonspecific miscellaneous systemic and gastro-enteric signs such as gagging, coughing, vomiting, oedema, anorexia, weight loss, stunted growth as well as decreased exercise tolerance are the most common clinical symptoms [1,22,23]. As described above, the definitive diagnosis requires demonstration of the first stage larvae in the faeces, tracheal wash or bronchoalveolar

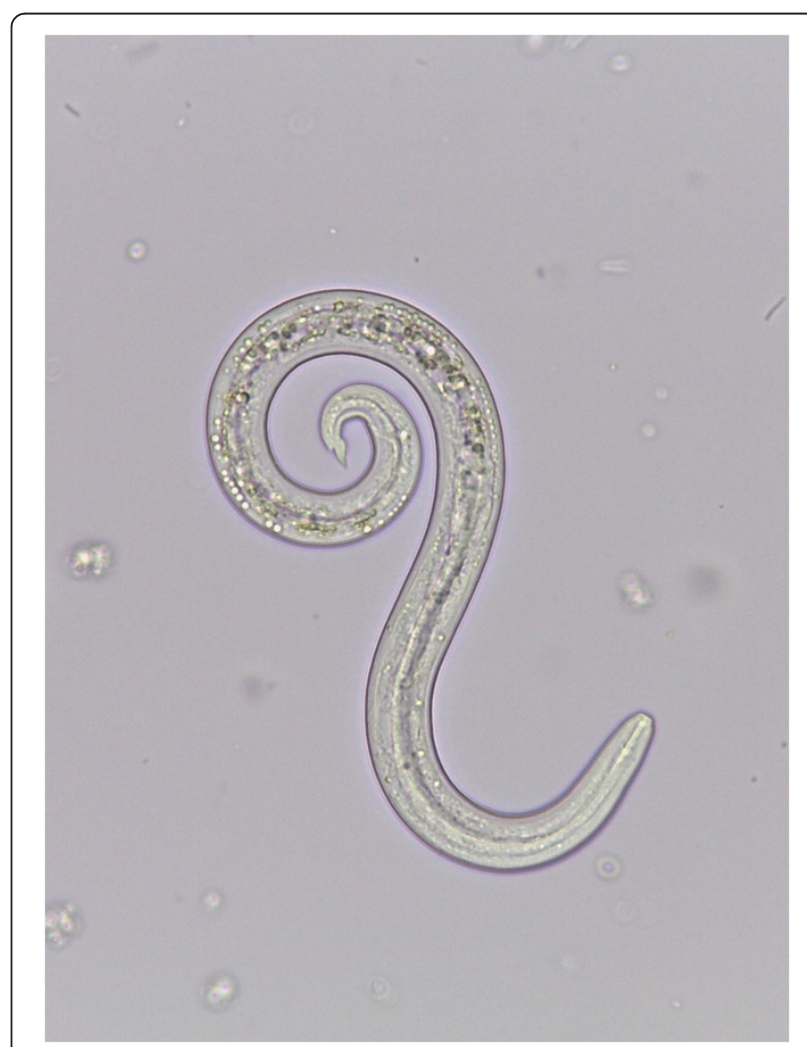

Figure 9 First-stage larva of Angiostrongylus vasorum. The L1 larvae when examined under a light microscope are approximately $350 \mu \mathrm{m}$ in length (range $310-400 \mu \mathrm{m}$ ), with a characteristic kinked tail and a dorsal spine and notch. 
lavage. Several case reports, including this one, show that faecal Baermann examination can be false negative, due to intermittent shedding of larvae, a high variation in the number of shedded larvae and the long pre-patent period [2]. Thus, examination of faecal samples from three consecutive days is recommended and tracheal wash should be considered [2].

The respiratory syndrome observed in the antemortem case is typical of that reported for A. vasorum infection in dog. The pulmonary lesions in dog angiostrongylosis are well described in the literature [24] and the present findings are in accordance with current knowledge [25]. The most consistent finding in our dog was interstitial pneumonia with prominent vascular changes. Pneumonia, in this case, was generally granulomatous with variable amounts of suppurative and eosinophilic inflammation. Small granulomatous foci of inflammation, associated with nematodes eggs and larvae, were found in many organs such as kidney, liver, pancreas. This is in accordance with the recent findings that showed the presence of the lesions in different organs [2], not only in the cardiovascular and respiratory systems. Thus, the absence of the typical signs should not preclude consideration of angiostrongylosis as a differential diagnosis [26]. The pathological findings in our case are in according with this propensity and should be considered during the diagnosis of suspected cases.

The findings of the present study confirm that the French heartworm $A$. vasorum should be considered in the differential diagnosis of pulmonary disease in dogs in Italy [27].

The results of this research demonstrated also the occurrence of A. vasorum L1 in fecal samples of dogs from the Campania region, as reported in other areas of central and southern Italy with an infection rate similar to that recently reported also in other studies [28-32]. In addition, they also show that FLOTAC can be utilized for diagnosis of $A$. vasorum infection, as already demonstrated for other lungworms as Crenosoma vulpis [33] in dogs and Aelurostrongylus abstrusus [34] in cats. Furthermore, given the lack of specificity of clinical signs, these infections are often not included in differential diagnosis, and animals remain infected and untreated. The FLOTAC has the advantage to be multivalent and therefore also other parasites (protozoa, nematoda, trematoda and cestoda) can be detected, which may be important in determining the cause of non-specific symptoms. A valid and affordable diagnostic method for the detection of $A$. vasorum infected animals before the appearance of clinical signs could avoid the onset of severe pathological changes in early anthelmintic-treated animals [35].

The emergence of $A$. vasorum as an important agent of respiratory disease in dogs, and apparent ongoing expansion of its range, must underline the need of an enhanced awareness concerning the knowledge of its epidemiology and biology [4].

\section{Ethics}

The animals used in the present study were sampled following approval by the animal ethics and welfare committee of the University of Naples Federico II (in Italian, Comitato Etico-scientifico per la Sperimentazione Animale dell' Università di Napoli Federico II; protocol number 0075262).

\section{Competing interest}

GC is the inventor of the FLOTAC apparatus. The method is licensed free of charge to universities and interested public non-commercial research centers. All other authors have no competing interest.

\section{Authors' contributions}

$L R, L C, S P-$ Participated in the study design and prepared the manuscript. LC and LM - Performed the clinical investigations. LR, PP and GC - Performed the parasitological investigations. TBP and SP - Carried out the histopatological investigations. GG and SP - Conceived the study and participated in its design and coordination. All authors read and approved the final manuscript.

\section{Acknowledgments}

The authors acknowledge Doctors Maria Paola Maurelli and Vincenzo Musella for their participation in the study.

Received: 22 April 2014 Accepted: 23 September 2014

Published online: 28 September 2014

\section{References}

1. Ferdushy T, Hasan MT: Angiostrongylus vasorum: the "French Heartworm". Parasitol Res 2010, 107:765-771.

2. Denk D, Matiasek K, Just FT, Hermanns W, Baiker K, Herbach N, Steinberg T, Fischer A: Disseminated angiostrongylosis with fatal cerebral haemorrhages in two dogs in Germany: a clinical case study. Vet Parasitol 2009, 160:100-108.

3. Morgan ER, Shaw S: Angiostrongylus vasorum infection in dogs: continuing spread and developments in diagnosis and treatment. J Small Anim Pract 2010, 51:616-621.

4. Morgan ER, Shaw SE, Brennan SF, de Waal TD, Jones BR, Mulcahy G: Angiostrongylus vasorum: a real heartbreaker. Trends Parasitol 2005, 21:49-51.

5. Conboy GA: Canine angiostrongylosis: the French heartworm: an emerging threat in North America. Vet Parasitol 2011, 176:382-389.

6. Morgan ER, Jefferies R, Krajewski M, Ward P, Shaw SE: Canine pulmonary angiostrongylosis: the influence of climate on parasite distribution. Parasitol Int 2009, 58:406-410.

7. Traversa D, Di Cesare A, Conboy G: Canine and feline cardiopulmonary parasitic nematodes in Europe: emerging and underestimated. Parasit Vectors 2010, 3:62.

8. Traversa D, Guglielmini C: Feline aelurostrongylosis and canine angiostrongylosis: a challenging diagnosis for two emerging verminous pneumonia infections. Vet Parasitol 2008, 157:163-174.

9. Gasser RB, Jabbar A, Mohandas N, Schnyder M, Deplazes P, Littlewood DTJ, Jex AR: Mitochondrial genome of Angiostrongylus vasorum: comparison with congeners and implications for studying the population genetics and epidemiology of this parasite. Infect Genet Evol 2012, 12:1884-1891.

10. Koch J, Willesen JL: Canine pulmonary angiostrongylosis: an update. Vet J 2009, 179:348-359.

11. Cringoli G, Rinaldi L, Maurelli MP, Utzinger J: FLOTAC: new multivalent techniques for qualitative and quantitative copromicroscopic diagnosis of parasites in animals and humans. Nat Protoc 2010, 5:503-515.

12. Schnyder M, Maurelli MP, Morgoglione ME, Kohler L, Deplazes $P$, Torgerson $P$, Cringoli G, Rinaldi L: Comparison of faecal techniques including FLOTAC for copromicroscopic detection of first stage larvae of Angiostrongylus vasorum. Parasitol Res 2011, 109:63-69.

13. Verzberger-Epshtein I, Markham RJ, Stryhn H, Sheppard JA, Whitney H, Conboy GA: Serologic detection of Angiostrongylus vasorum infection in dogs. Vet Parasitol 2008, 151:53-60. 
14. Schnyder M, Stebler K, Naucke TJ, Lorentz S, Deplazes P: Evaluation of a rapid device for serological in-clinic diagnosis of canine angiostrongylosis. Parasit Vectors 2014, 7:72.

15. Al-Sabi MN, Deplazes P, Webster P, Willesen JL, Davidson RK, Kapel CM: PCR detection of Angiostrongylus vasorum in faecal samples of dogs and foxes. Parasitol Res 2010, 107:135-140.

16. Jefferies R, Morgan ER, Helm J, Robinson M, Shaw SE: Improved detection of canine Angiostrongylus vasorum infection using real-time PCR and indirect ELISA. Parasitol Res 2011, 109:1577-1583.

17. Hendrix CM: Diagnostic Veterinary Parasitology. 2nd edition. St. Louis: Mosby; 1998.

18. Rosen $L$, Ash $L R$, Wallace GD: Life history of canine lungworm Angiostrongylus vasorum (Baillet). Am J Vet Res 1970, 31:131-141.

19. Helm JR, Morgan ER, Jackson MW, Wotton P, Bell R: Canine angiostrongylosis: an emerging disease in Europe. J Vet Emerg Crit Care (San Antonio) 2010, 20:98-109.

20. Lepri E, Veronesi F, Traversa D, Conti MB, Marchesi MC, Miglio A, Mandara MT: Disseminated angiostrongylosis with massive cardiac and cerebral involvement in a dog from Italy. Parasitol Res 2011, 109:505-550.

21. Zarelli M, Shiel R, Gallagher B, Skelly C, Cahalan S: Imaging diagnosis: CT findings in a dog with intracranial hemorrhage secondary to angiostrongylosis. Vet Radiol Ultrasound 2012, 53:420-423.

22. Chapman PS, Boag AK, Guitian J, Boswood A: Angiostrongylus vasorum infection in 23 dogs (1999-2002). J Small Anim Pract 2004, 45:435-440.

23. Traversa D, Di Cesare A, Meloni S, Frangipane Di Regalbono A, Milillo P, PAMPURINI F, Venco L: Canine angiostrongylosis in Italy: occurrence of Angiostrongylus vasorum in dogs with compatible clinical pictures. Parasitol Res 2013, 112:2473-2480

24. Yamakawa Y, Mcgarry JW, Denk D, Dukes-Mcewan J, Macdonald N, MAS A, Mcconnell F, Tatton B, Valentine EG, Wayne J, Williams JM, Hetzel U: Emerging canine angiostrongylosis in Northern England: five fatal cases. Vet Rec 2009, 164:149-152.

25. Bolt G, Monrad J, Koch J, Jensen AL: Canine angiostrongylosis: a review. Vet Rec 1994, 135:447-452.

26. Gallagher B, Brennan SF, Zarelli M, Mooney CT: Geographical, clinical, clinicopathological and radiographic features of canine angiostrongylosis in Irish dogs: a retrospective study. Ir Vet J 2012, 65:5.

27. Traversa D, Torbidone A, Malatesta D, Guglielmini C: Occurrence of fatal canine Angiostrongylus vasorum infection in Italy. Vet Parasitol 2008, 152:162-166.

28. Sasanelli M, Paradies P, Otranto D, Lia RP, de Caprariis D: Haemothorax associated with Angiostrongylus vasorum infection in a dog. I Small Anim Pract 2008, 49:417-420.

29. Di Cesare A, Castagna G, Meloni S, Milillo P, Latrofa S, Otranto D, Traversa D: Canine and feline infections by cardiopulmonary nematodes in Central and Southern Italy. Parasitol Res 2011, 109:S87-S96.

30. Tieri E, Pomilio F, Di Francesco G, Saletti MA, Totaro P, Troilo M, Menna S, Tampieri MP, Morelli D: Angiostrongylus vasorum in 20 dogs in the province of Chieti, Italy. Vet Ital 2011, 47:77-88.

31. Guardone L, Schnyder M, Macchioni F, Deplazes P, Magi M: Serological detection of circulating Angiostrongylus vasorum antigen and specific antibodies in dogs from Central and Northern Italy. Vet Parasitol 2013, 192:192-198.

32. Pipia AP, Varcasia A, Tosciri G, Seu S, Manunta ML, Mura MC, Sanna G, Tamponi C, Brianti E, Scala A: New insights onto cardiopulmonary nematodes of dogs in Sardinia, Italy. Parasitol Res 2014, 113:1505-1509.

33. Rinaldi L, Calabria G, Carbone S, Carrella A, Cringoli G: Crenosoma vulpis in dog: first case report in Italy and use of the FLOTAC technique for copromicroscopic diagnosis. Parasitol Res 2007, 101:1681-1684.

34. Gaglio G, Cringoli G, Rinaldi L, Brianti E, Giannetto S: Use of the FLOTAC technique for the diagnosis of Aelurostrongylus abstrusus in the cat. Parasitol Res 2008, 103:1055-1057.

35. Schnyder M, Tanner I, Webster P, Barutzki D, Deplazes P: An ELISA for sensitive and specific detection of circulating antigen of Angiostrongylus vasorum in serum samples of naturally and experimentally infected dogs. Vet Parasitol 2011, 179:152-158.

\section{Submit your next manuscript to BioMed Central and take full advantage of:}

- Convenient online submission

- Thorough peer review

- No space constraints or color figure charges

- Immediate publication on acceptance

- Inclusion in PubMed, CAS, Scopus and Google Scholar

- Research which is freely available for redistribution 